\title{
Statistical translation invariance protects a topological insulator from interactions
}

\author{
A. Milsted, ${ }^{1}$ L. Seabra, ${ }^{2}$ I. C. Fulga ${ }^{3}$ C. W. J. Beenakker, ${ }^{4}$ and E. Cobanera ${ }^{5}$ \\ ${ }^{1}$ Institut für Theoretische Physik, Leibniz Universitat Hannover, Appelstrasse 2, 30167 Hannover, Germany \\ ${ }^{2}$ Department of Physics, Technion - Israel Institute of Technology, Haifa 32000, Israel \\ ${ }^{3}$ Department of Condensed Matter Physics, Weizmann Institute of Science, Rehovot 76100, Israel \\ ${ }^{4}$ Instituut-Lorentz, Universiteit Leiden, P.O. Box 9506, 2300 RA Leiden, The Netherlands \\ ${ }^{5}$ Institute for Theoretical Physics, Center for Extreme Matter and Emergent Phenomena, Utrecht University, Leuvenlaan 4, \\ 3584 CE Utrecht, The Netherlands \\ (Received 27 April 2015; published 24 August 2015)
}

\begin{abstract}
We investigate the effect of interactions on the stability of a disordered, two-dimensional topological insulator realized as an array of nanowires or chains of magnetic atoms on a superconducting substrate. The Majorana zero-energy modes present at the ends of the wires overlap, forming a dispersive edge mode with thermal conductance determined by the central charge $c$ of the low-energy effective field theory of the edge. We show numerically that, in the presence of disorder, the $c=1 / 2$ Majorana edge mode remains delocalized up to extremely strong attractive interactions, while repulsive interactions drive a transition to a $c=3 / 2$ edge phase localized by disorder. The absence of localization for strong attractive interactions is explained by a self-duality symmetry of the statistical ensemble of disorder configurations and of the edge interactions, originating from translation invariance on the length scale of the underlying mesoscopic array.
\end{abstract}

DOI: 10.1103/PhysRevB.92.085139 PACS number(s): 73.20.Fz, 03.65.Vf, 71.20.-b, 74.78.Na

\section{INTRODUCTION}

In the very first topological insulator ever discovered, the quantum Hall insulator [1], interactions have a dramatic effect by changing the quantum of conductance from the value $e^{2} / h$ to a rational fraction of it [2]. The quantum Hall insulator is but one entry in a periodic table of topological states of matter, including both insulators and superconductors (and commonly referred to as topological insulators or TIs) [3-6]. These materials are all characterized by a gapped bulk with gapless surface or edge excitations, protected from localization by disorder due the existence of a topological invariant.

Because a topological invariant is a property of the singleparticle Hamiltonian, it is a challenge to classify TIs in the presence of interactions [7-11], and in some cases it is even possible to show explicitly that the topological classification is unstable to interactions [12]. The key questions are whether interactions may localize the surface states or lead to distinct topological phases, as they do in the fractional quantum Hall effect. Here we address these two questions for the superconducting counterpart of the quantum Hall insulator, a twodimensional (2D) superconductor with chiral $p$-wave pairing.

The superconducting analog of the electronic quantum Hall effect, the thermal quantum Hall effect, refers to a heat current carried by Majorana modes propagating along the edge of a topological superconductor [13-15]. In the absence of backscattering along the edge, the thermal conductance equals $G_{\text {thermal }}=c G_{0}$, with $G_{0}=\pi^{2} k_{\mathrm{B}}^{2} T / 3 h$ the thermal conductance quantum for free electrons. The coefficient $c$, called the central charge, characterizes the stress-energy tensor of the conformal field theory associated with the low-energy edge modes [14,16-19]. While $c=1$ for the Luttinger edge mode of the integer and fractional quantum Hall effects, a Majorana edge mode has $c=1 / 2$ - at least in the absence of interactions [14]. One of our discoveries is that moderate repulsive interactions between the Majorana fermions drive a transition to an extended $c=3 / 2$ edge phase.
Chiral $p$-wave superconductors may exist naturally $\left(\mathrm{Sr}_{2} \mathrm{RuO}_{4}\right.$ is a candidate material $\left.[20,21]\right)$, but a major recent development is the search for this particular exotic pairing in semiconductor nanowires [22-25] and chains of magnetic atoms [26] deposited on a superconductor with conventional $s$-wave pairing. (Alternative proposals include chains of atoms in optical lattices [27] and chains of magnetic vortices on the surface of a three-dimensional (3D) TI [28]). A twodimensional (2D) array of parallel nanowires, see Fig. 1, forms an anisotropic topological insulator, called a "weak" TI [29-36] because the Majorana mode propagates only along the edges perpendicular to the wires. Each nanowire realizes a Kitaev chain [37], with two unpaired Majorana zero-energy modes at the end points of the wire. These zero modes overlap to form a dispersive one-dimensional (1D) edge mode of Majorana fermions [38-40]. Backscattering by disorder is not forbidden, yet this "Kitaev edge mode" does not localize [41,42].

Because the effective boundary theory of the Kitaev edge is one dimensional, it is possible to investigate its behavior in great detail with modern numerical tools based on matrix product state (MPS) methods [we use both variational infinite MPS methods and the density-matrix renormalization group (DMRG)]. The average translation symmetry of the mesoscopic array causes a statistical translation symmetry of the ensemble of disorder configurations and the exact self-dual structure of the interacting edge Hamiltonian. This situation is an example of a "statistical topological insulator," protected by a symmetry that is present only on average [41]. We find that the exact self-dual structure of the interaction Hamiltonian protects the gapless Majorana mode up to extremely strong attractive interactions. A gapped phase does appear for repulsive interactions, but first the Kitaev edge enters a gapless phase with an unusually large central charge $c=3 / 2$.

Our findings are consistent with two very recent and independent investigations of the same model in the clean limit by Rahmani et al. $[43,44]$. 


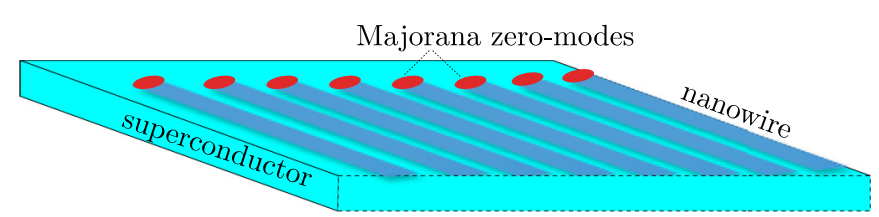

FIG. 1. (Color online) Array of nanowires on a superconducting substrate, with a delocalized Majorana edge mode composed out of coupled zero modes localized at the end points.

The paper is organized as follows. In Sec. II we introduce a minimal model of the Kitaev edge for generic interactions in the host 2D superconductor, as a necessarily self-dual, interacting Majorana chain. The Jordan-Wigner transformation maps the model to a spin- $\frac{1}{2}$ chain. In Sec. III we discuss the phase diagram of this model as a function of the relative strength of interactions to hopping, and extract the central charges of its critical phases. In Sec. IV we consider the effect of disorder on those critical phases to assess the stability of transport measurements. We conclude in Sec. V.

\section{INTERACTING KITAEV EDGE}

The end points of each nanowire (labeled $s=1,2, \ldots$ ) in the array of Fig. 1 form a 1D lattice of self-conjugate Majorana operators $\left(\gamma_{s}=\gamma_{s}^{\dagger}\right)$, governed by the Hamiltonian

$$
H=-i \sum_{s} \alpha_{s} \gamma_{s} \gamma_{s+1}-\sum_{s} \kappa_{s} \gamma_{s} \gamma_{s+1} \gamma_{s+2} \gamma_{s+3}
$$

The $\alpha_{s}$ terms describe hopping along the edge and the $\kappa_{s}$ terms describe interactions.

To interpret this Hamiltonian it is helpful to transform pairs of Majorana operators into creation and annihilation operators of effective edge fermions,

$$
\gamma_{2 j-1}=c_{j}+c_{j}^{\dagger}, \quad i \gamma_{2 j}=c_{j}-c_{j}^{\dagger} .
$$

For odd $s$ the four-Majorana terms have the interpretation of density-density interactions [28], since

$$
-\gamma_{s} \gamma_{s+1} \gamma_{s+2} \gamma_{s+3}=\left(2 n_{j}-1\right)\left(2 n_{j+1}-1\right)
$$

for $s=2 j-1$. The appearance of an interaction term with $s=2 j$ even,

$$
\begin{aligned}
& -\gamma_{s} \gamma_{s+1} \gamma_{s+2} \gamma_{s+3} \\
& \quad=-i\left(c_{j}-c_{j}^{\dagger}\right)\left(2 n_{j+1}-1\right)\left(c_{j+2}+c_{j+2}^{\dagger}\right),
\end{aligned}
$$

is then dictated by translation symmetry. The presence of this term is what distinguishes the Kitaev chain (a 1D array) from the Kitaev edge (the 1D edge of a 2D array) [42].

The fact that all two- and four-Majorana terms appear on equal footing, that is, there is no statistical distinction between $\alpha_{2 j-1}, \kappa_{2 j-1}$ and $\alpha_{2 j}, \kappa_{2 j}$ determines that the edge Hamiltonian is exactly self-dual in the sense of quantum statistical mechanics [45]. The apparent "dimerization" of the Kitaev edge introduced by the transformation to effective fermions is fictitious: In contrast to the Kitaev chain, here there is no physical distinction between even and odd sites. This symmetry is enforced by the bulk origin of the edge theory.
In what follows we analyze the model Hamiltonian (1) because it is the simplest Hamiltonian compatible with translation symmetry. It may be thought of as the leading term in an expansion of the actual, microscopic in origin, interaction. It is not possible to take Eqs. (3) and (4) as a direct indication of what the microscopic interaction may be, since the effective $c$ fermions are complicated combinations of the microscopic fermions.

We perform a Jordan-Wigner transformation to an equivalent spin- $\frac{1}{2}$ representation, by writing the Majorana operators in terms of Pauli matrices:

$$
\gamma_{2 k}=\sigma_{k}^{y} \prod_{j=1}^{k-1} \sigma_{j}^{z}, \quad \gamma_{2 k-1}=\sigma_{k}^{x} \prod_{j=1}^{k-1} \sigma_{j}^{z} .
$$

This transformation splits the coupling parameters into even, $\alpha_{s}^{\mathrm{e}} \equiv \alpha_{2 s}, \kappa_{s}^{\mathrm{e}} \equiv \kappa_{2 s}$, and odd, $\alpha_{s}^{\mathrm{o}} \equiv \alpha_{2 s+1}, \kappa_{s}^{\mathrm{o}} \equiv \kappa_{2 s+1}$, sets. Statistical translation invariance dictates that the even and odd sets are indistinguishable in a clean system and have the same probability distribution in a disordered ensemble.

The Hamiltonian (1) of the interacting Kitaev edge transforms into

$$
\begin{aligned}
H= & -\sum_{s} \alpha_{s}^{\mathrm{e}} \sigma_{s}^{x}-\sum_{s} \alpha_{s}^{\mathrm{o}} \sigma_{s}^{z} \sigma_{s+1}^{z} \\
& +\sum_{s} \kappa_{s}^{\mathrm{e}} \sigma_{s}^{x} \sigma_{s+1}^{x}+\sum_{s} \kappa_{s}^{\mathrm{o}} \sigma_{s}^{z} \sigma_{s+2}^{z} .
\end{aligned}
$$

This spin model is the self-dual anisotropic next-nearestneighbor Ising (ANNNI) model. The standard $\left(\kappa_{s}^{\mathrm{e}}=0\right)$ ANNNI model $[46,47]$ has generically a gapped spectrum, except for a critical line with $c=0.5$ and a floating phase with $c=1$. (See Refs. [48,49] for a recent study in the context of Majorana zero modes).

The special feature of the Kitaev edge that protects the gapless phase is the equivalence of the even and odd coupling terms $[41,42]$. The ANNNI Hamiltonian (6) satisfies a corresponding self-duality relation [45], which in the context of a spin- $\frac{1}{2}$ chain would require an artificial fine tuning of parameters. Here the self-duality is inherited from the realization of the Kitaev edge Hamiltonian (1), where it expresses the natural requirement that the translation of the Majorana operators by one site, $\gamma_{s} \mapsto \gamma_{s+1}$, should describe the same physical system. Self-duality pins the Kitaev edge at a gapless critical point between two gapped phases, protecting it from localization by disorder or finite but potentially extremely large interactions.

\section{PHASE DIAGRAM OF THE CLEAN EDGE}

In the absence of disorder we may set $\kappa_{s}^{\mathrm{e}}=\kappa_{s}^{\mathrm{o}} \equiv \kappa$ and $\alpha_{s}^{\mathrm{e}}=\alpha_{s}^{\mathrm{o}} \equiv \alpha$ ( $>0$ for definiteness [50]). Then the edge is controlled by only one dimensionless interaction-strength parameter $\zeta=\kappa / \alpha$.

We examine the phase diagram of the clean Kitaev edge using the evoMPS implementation [51-53] of the MPS timedependent variational principle and conjugate gradient solver in the thermodynamic limit. To determine the central charge of the gapless (critical) phases we divide the infinite chain into half-chains $A$ and $B$ and calculate the entanglement 


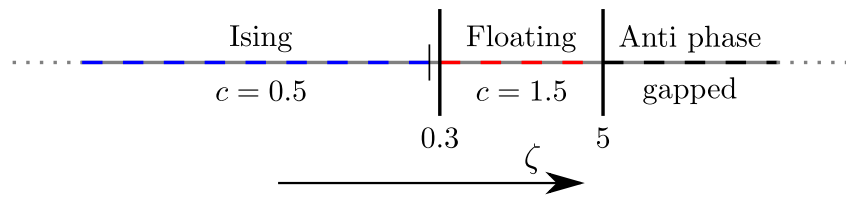

FIG. 2. (Color online) Quantum phase diagram of the interacting Kitaev edge as a function of the relative strength $\zeta$ of interactions and hopping along the edge. The central charges associated with the gapless phases are indicated.

entropy

$$
S=-\operatorname{Tr} \rho_{A} \ln \rho_{A}
$$

from the reduced density matrix $\rho_{A}$. The scaling

$$
S=\frac{1}{6} c \ln \xi+\text { const. }
$$

of $S$ with the correlation length $\xi$ of the slowest decaying correlation function provides an estimate of the central charge [54-56]. A saturation of $S$ with $\xi$ on the other hand provides strong evidence for a gapped phase. In this way we obtain the three phases indicated in Fig. 2. Representative numerical data for the floating phase are shown in Fig. 3.

The noninteracting point $\zeta=0$ corresponds to the critical phase of the Ising model, with central charge $c=1 / 2$. We find that this phase persists up to attractive interactions that are very large compared to mesoscopic energy scales, but ultimately it will break down. Our numerics extends up to $\zeta=-100$. Rahmani et al. [43] report a phase transition into a gapped phase for $\zeta \approx-250$.

In order to analyze the effect of very strong interactions we investigate the singular limit $\zeta \rightarrow \pm \infty$, when the Hamiltonian contains only the interaction term,

$$
\lim _{\zeta \rightarrow \pm \infty} H= \pm \sum_{s} \gamma_{s} \gamma_{s+1} \gamma_{s+2} \gamma_{s+3} .
$$

The two signs are connected by a gauge transformation (but only if $\alpha=0$ ), so we focus on the positive sign. To assess the role of translation invariance, we have found it helpful to study

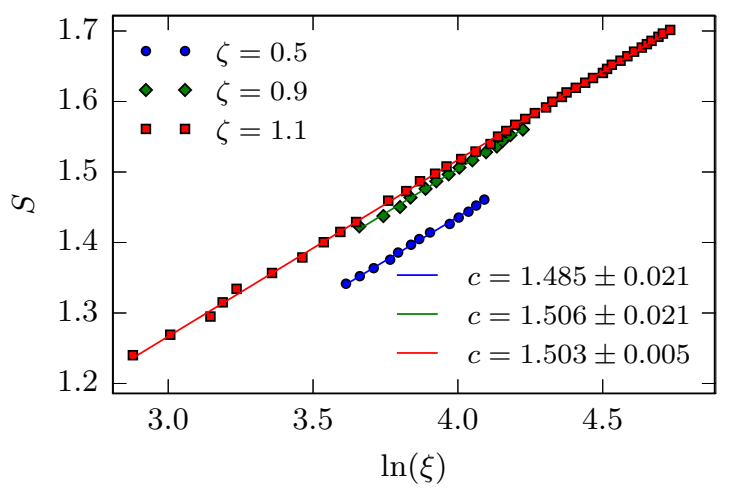

FIG. 3. (Color online) Correlation length dependence of the entanglement entropy for different control parameters $\zeta$ in the "floating" phase. The straight line fits to $S=(c / 6) \ln \xi+$ const. indicate a central charge of $c=3 / 2$. Bond dimensions used are in the range $18 \leqslant D \leqslant 102$. All states were converged up to an effective energy gradient norm $\leqslant 10^{-8}$.
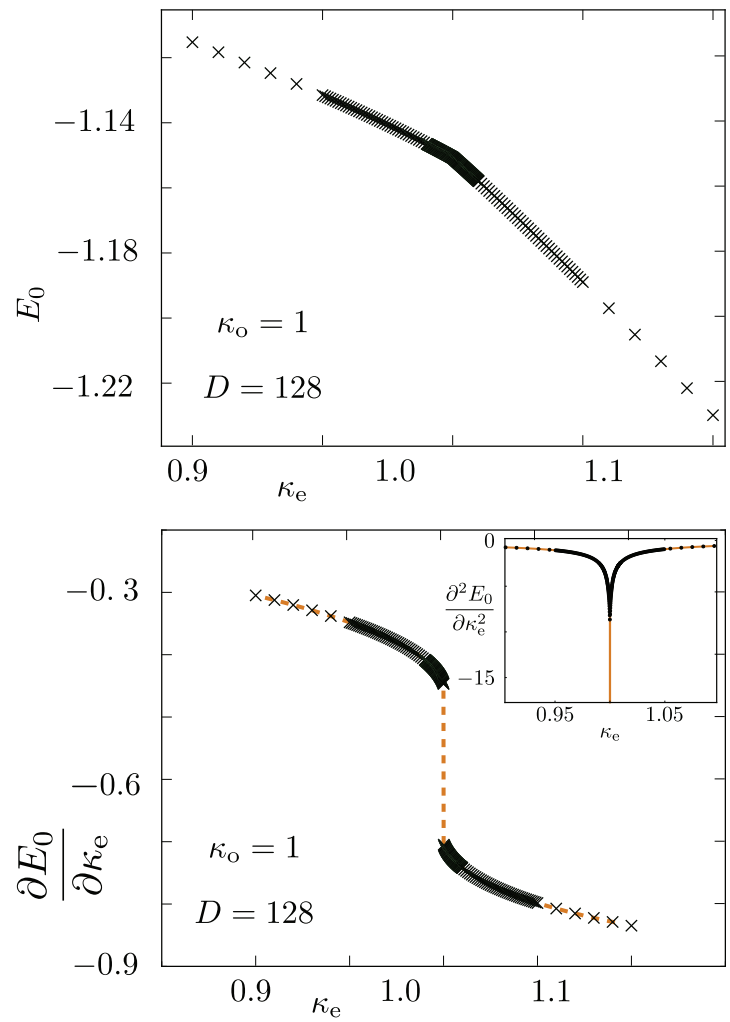

FIG. 4. (Color online) Ground state energy (top panel), as well as its first and second derivatives (bottom panel, inset of bottom panel) as a function of $\kappa_{e}$. We have used a constant $\kappa_{\mathrm{o}}=1$ and bond dimension $D=128$ throughout. The first derivative is discontinuous across the phase transition (dashed line).

the Hamiltonian

$$
\begin{aligned}
H= & \kappa_{\mathrm{e}} \sum_{s=2 k} \gamma_{s} \gamma_{s+1} \gamma_{s+2} \gamma_{s+3} \\
& +\kappa_{\mathrm{o}} \sum_{s=2 k-1} \gamma_{s} \gamma_{s+1} \gamma_{s+2} \gamma_{s+3}
\end{aligned}
$$

as a function of $\kappa_{\mathrm{e}}$ around the self-dual point $\kappa_{\mathrm{e}}=\kappa_{\mathrm{o}} \equiv 1$.

Figure 4 shows the behavior of the ground state energy, as well as its first and second derivatives, as $\kappa_{\mathrm{e}}$ is varied through the self-dual point. The first derivative is discontinuous at the phase transition. To further confirm that the transition at $\kappa_{e}=1$ is of first order, we have performed scaling of the energy gap as a function of bond dimension. The results of Fig. 5 show that the gap remains finite in the thermodynamic limit, in agreement with the entanglement entropy. The same behavior was reported recently in Ref. [43].

Since the Kitaev edge is gapped in the limit $\zeta \rightarrow \infty$, it is natural to ask whether this phase of infinitely strong interactions is adiabatically connected to the gapped antiphase that we find for $\zeta>5$. In order to address this question, we investigated the Kitaev edge for $\zeta^{-1} \geqslant 0$. We conclude that the antiphase does indeed persist all the way to $\zeta \rightarrow \infty$, and that there is no transition in the singular limit. This situation should be contrasted with what happens for $\zeta \rightarrow-\infty$.

For $\zeta>0$ a second-order phase transition takes place at $\zeta \approx 0.3$, leading to a new gapless phase characterized 


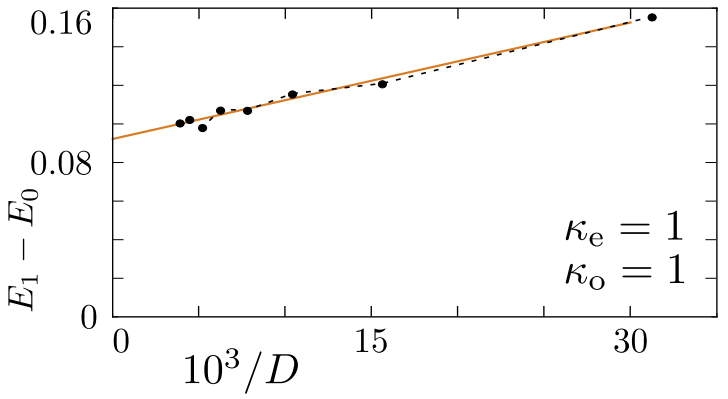

FIG. 5. (Color online) Energy difference between the ground state and the first excited level as a function of inverse bond dimension $1 / D$ at the self-dual point $\kappa_{\mathrm{e}}=\kappa_{\mathrm{o}}=1$. The system gap scales to a value $E_{1}-E_{0} \simeq 0.092$ as the bond dimension is extrapolated to infinity, indicating that the self-dual point is gapped in the thermodynamic limit.

by incommensurate charge-density waves. We examine the dominant wave vector $k$ corresponding to modulations in the connected $\sigma^{x}$ and $\sigma^{z}$ spin-spin correlation functions (see Fig. 6). In the Ising phase $(\zeta \lesssim 0.3)$ these correlation functions have diverging correlation length and no modulations, so $k=0$. At $\zeta \approx 0.3$ the system enters a "floating" phase in which the wave vector varies continuously. Unlike the floating phase of the standard ANNNI model, the floating phase of the Kitaev edge has has an unusually large central charge of $c=3 / 2$, see Fig. 3. This transition into a phase with coexisting
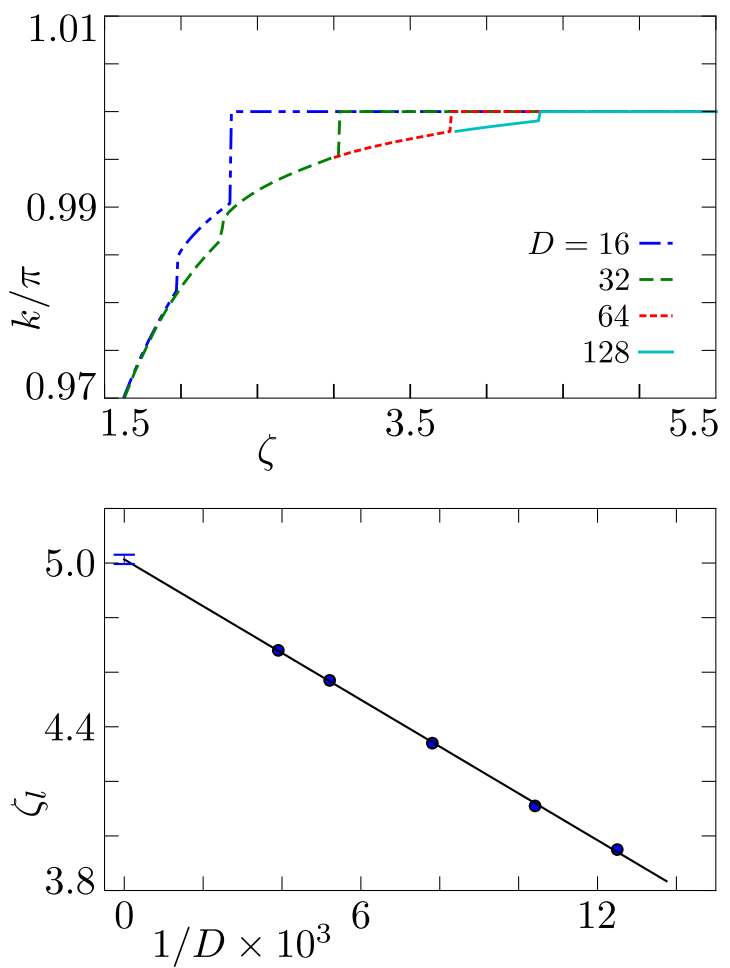

FIG. 6. (Color online) Top panel: Locking of the wave vector corresponding to $\sigma^{x}$ modulations as a function of the control parameter $\zeta$ for different bond dimensions $D$. Bottom panel: Extrapolation of the locking point $\zeta_{l}$ to infinite bond dimension.
Luttinger $(c=1)$ and Majorana $(c=1 / 2)$ edge modes has been confirmed by Rahmani et al. [44].

For sufficiently large $\zeta \gtrsim 5$, the wave vector locks to a commensurate value: $k=\pi / 2$ for $\sigma^{z}$ and $k=\pi$ for $\sigma^{x}$. A finite discontinuity in the second derivative of the energy accompanies the locking of the wave vector. We interpret the locking of the wave vector as a transition into a phase characterized by a commensurate charge-density wave, akin to the gapped antiphase of the standard ANNNI model. The value of $\zeta$ where the locking takes place depends significantly on the size of our numerical simulation (more precisely, on the bond dimension $D$ of the infinite MPS), and therefore the transition point at $\zeta \approx 5$ is a $D \rightarrow \infty$ extrapolation, as shown in Fig. 6.

\section{EFFECT OF DISORDER}

We model disorder in the Kitaev edge by shifting the nearest-neighbor hopping terms $\alpha_{s}^{\mathrm{o}}$ and $\alpha_{s}^{\mathrm{e}}$ by a random amount in the range $[-\delta, \delta]$, drawn independently and uniformly for each lattice site. Because $\alpha_{s}^{\mathrm{o}}$ and $\alpha_{s}^{\mathrm{e}}$ are statistically equivalent, the translation invariance on long length scales is not broken by disorder. The disorder-averaged entanglement entropy $\mathcal{S}$ of a delocalized 1D system with open boundaries (divided into segments of length $x$ and $L-x$ ) is given by [57-59]

$$
\mathcal{S}(x)=\frac{1}{6} \widetilde{c} \ln [(2 L / \pi) \sin (\pi x / L)]+\text { const. }
$$

This formula generalizes the clean-system relation (8), with $\widetilde{c}$ an effective central charge instead of the usual central charge $c$ associated with translation-invariant systems.

Previous work [57,59] has shown that the addition of disorder to a critical Ising phase $(c=1 / 2)$ drives the spin system to a gapless phase of random spin singlets, each contributing $\ln 2$ to the entanglement entropy. Characteristic signatures of this phase are

(1) An effective central charge $\tilde{c}=\frac{1}{2} \ln 2 \approx 0.347$.

(2) The appearance of a peak at $\ln 2$ in the probability distribution of $\mathcal{S}$ due to the singlet contribution.

In Fig. 7 we search for these signatures, both in the $c=1 / 2$ Ising phase and in the $c=3 / 2$ floating phase, using an MPS implementation of the DMRG method [60,61].

For the Kitaev edge at $\zeta=0$ we find that the effective central charge converges quickly to $\widetilde{c}=\frac{1}{2} \ln 2$, as expected [green data points in Fig. 7(a)]. For small values of $\zeta<0$, we also observe the same behavior [blue data points in Fig. 7(a)] very clearly. The fact that the Kitaev edge remains delocalized for small $\zeta<0$, or equivalently, that the self-dual ANNNI model remains in the random spin-singlet phase, is further confirmed by the narrow peak developing at $\ln 2$ in the halfchain entropy distribution, see Fig. 7(b). Finite-size corrections become more and more significant with increasing $-\zeta$, making it difficult to reach a good convergence for $\zeta=-1$. The red data points in Fig. 7(a) give a value of $\widetilde{c}$ which we believe has not yet fully converged for $L=300$, but still seems consistent with a delocalized edge.

Simulations in the floating phase $(0.3<\zeta<5.0)$ are computationally more expensive since the entanglement entropy, and therefore the required MPS bond dimension, is larger in this phase (keeping $L$ fixed) due to the unusually large central charge $c=1.5$. Moreover, in order to avoid incommensurate 
(a)

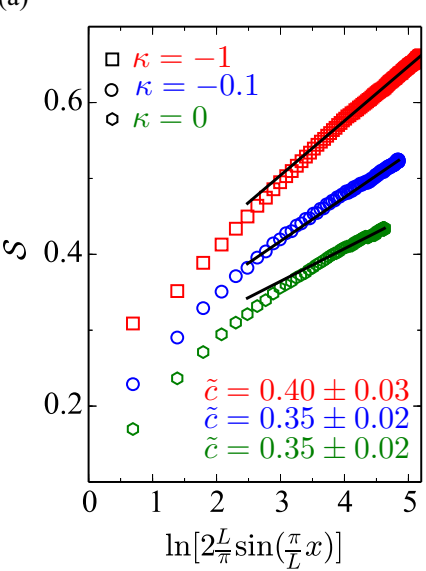

(c)

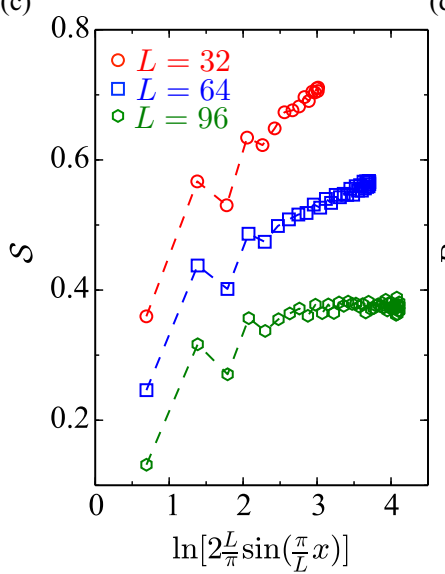

(b)

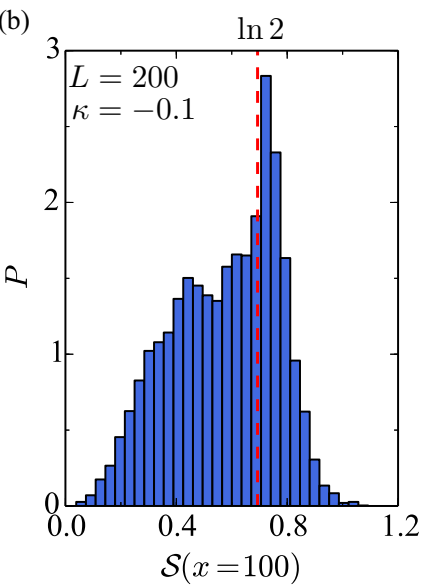

(d)

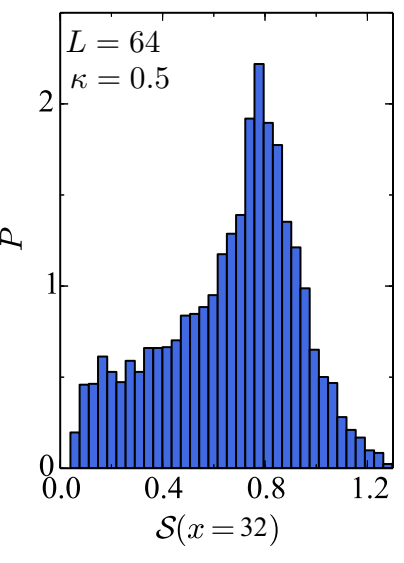

FIG. 7. (Color online) Top panels: Effect of disorder on the Ising phase at $\alpha=1$ with $\delta=0.5$. (a) Scaling of the average entanglement entropy for attractive interactions $\kappa=0,-0.1,-1$ at $L=100,200,300$, respectively, according to Eq. (11). The effective central charge is obtained from the slope of linear fits (solid lines) in the limit $x \rightarrow L / 2$ as $\tilde{c} / 6$. Data has been shifted vertically for clarity. (b) Normalized probability distribution of the half-chain entanglement entropy for $\kappa=-0.1$, showing a developing narrow singlet peak at $\ln 2$. Bottom panels: Effect of disorder on the floating phase at $\alpha=1, \delta=0.5$, and repulsive interactions $\kappa=0.5$. (c) The average entanglement entropy for $L=32,64,96$ saturates for large values of the scaling function. Lines are guides to the eye and the data have been shifted vertically for clarity. (d) Normalized probability distribution of the half-chain entanglement entropy for $L=64$, showing evidence for states with entanglement entropy $\mathcal{S} \rightarrow 0$. Up to $\sim 8000$ disorder realizations were employed for each simulation.

spin-spin correlations, it becomes necessary to select values of $\kappa$ which give a family of system sizes $L$ commensurate with the ground state wave vector. Figure 7(c) shows that the disorder-averaged entanglement entropy saturates as the

middle of the chain is approached for the largest system sizes available, indicating a gapped phase, albeit with a large value of $\mathcal{S}$. The probability distribution of Fig. 7(d) also shows a finite weight of the distribution for vanishingly small values of the average entropy, which is another indication of a gapped phase. We conclude that the floating phase is localized by disorder, most likely due to the pinning of incommensurate charge-density waves by the random spatial fluctuations of the disorder potential.

\section{CONCLUSIONS}

We have investigated the edge theory of an interacting topological insulator stabilized at criticality by a symmetry that is broken locally but restored on average. Such a 1D statistical topological insulator may be realized at the edge of an anisotropic $p$-wave superconductor. Of particular interest is the possibility of realizing the interacting Majorana edge mode studied here starting from a one-dimensional array of vortex lines [28], since this could produce larger interaction strengths than proposals involving semiconducting nanowires or atomic chains proximity coupled to superconductors.

Unlike typical one-dimensional models, the effective edge theory of the system remains critical, even for large attractive interaction strength and/or disorder strength. This behavior can be traced back to the average translation symmetry of the two-dimensional bulk, which imposes an average self-duality on the strongly interacting Kitaev edge.

We hope that our work will motivate the search for other strongly interacting topological phases in which average symmetries of the lattice lead to boundaries which remain delocalized in the presence of disorder.

\section{ACKNOWLEDGMENTS}

We thank A. R. Akhmerov, E. Altman, P. Fendley, M. Maksymenko, Y. Nakata, Z. Nussinov, J. Ruhman, N. Shannon, and T. J. Osborne for stimulating discussions. This work is part of the DITP consortium, a program of the Netherlands Organisation for Scientific Research (NWO/OCW). I.C.F. thanks the European Research Council under the European Union's Seventh Framework Programme (FP7/20072013)/ERC Project MUNATOP, the US-Israel Binational Science Foundation, and the Minerva Foundation for support. A.M. was supported by the ERC Grants QFTCMPS and SIQS and by the cluster of excellence EXC 201 Quantum Engineering and Space-Time Research. L.S. was supported by an Ali Kaufmann fellowship and the US-Israel Bi-National Science Foundation. C.B. is supported by an ERC Synergy grant.
[1] K. v. Klitzing, G. Dorda, and M. Pepper, Phys. Rev. Lett. 45, 494 (1980).

[2] H. L. Stormer, D. C. Tsui, and A. C. Gossard, Rev. Mod. Phys. 71, S298 (1999).
[3] A. Y. Kitaev, AIP Conf. Proc. 1134, 22 (2009).

[4] A. P. Schnyder, S. Ryu, A. Furusaki, and A. W. W. Ludwig, AIP Conf. Proc. 1134, 10 (2009).

[5] M. Z. Hasan and C. L. Kane, Rev. Mod. Phys. 82, 3045 (2010). 
[6] X.-L. Qi and S.-C. Zhang, Rev. Mod. Phys. 83, 1057 (2011).

[7] C.-X. Liu, X.-L. Qi, and S.-C. Zhang, Physica E 44, 906 (2012).

[8] X. Chen, Z.-C. Gu, Z.-X. Liu, and X.-G. Wen, Science 338, 1604 (2012).

[9] X. Chen, Z.-C. Gu, Z.-X. Liu, and X.-G. Wen, Phys. Rev. B 87, 155114 (2013).

[10] B. van Heck, E. Cobanera, J. Ulrich, and F. Hassler, Phys. Rev. B 89, 165416 (2014).

[11] T. Senthil, Annual Review of Condensed Matter Physics 6, 299 (2015).

[12] L. Fidkowski and A. Kitaev, Phys. Rev. B 83, 075103 (2011).

[13] T. Senthil and M. P. A. Fisher, Phys. Rev. B 61, 9690 (2000).

[14] N. Read and D. Green, Phys. Rev. B 61, 10267 (2000).

[15] A. Vishwanath, Phys. Rev. Lett. 87, 217004 (2001).

[16] H. W. J. Blöte, J. L. Cardy, and M. P. Nightingale, Phys. Rev. Lett. 56, 742 (1986).

[17] I. Affleck, Phys. Rev. Lett. 56, 746 (1986).

[18] H. Sumiyoshi and S. Fujimoto, J. Phys. Soc. Jpn. 82, 023602 (2013).

[19] B. Bradlyn and N. Read, Phys. Rev. B 91, 125303 (2015).

[20] A. P. Mackenzie and Y. Maeno, Rev. Mod. Phys. 75, 657 (2003).

[21] C. Kallin, Rep. Prog. Phys. 75, 042501 (2012).

[22] V. Mourik, K. Zuo, S. M. Frolov, S. R. Plissard, E. P. A. M. Bakkers, and L. P. Kouwenhoven, Science 336, 1003 (2012).

[23] M. T. Deng, C. L. Yu, G. Y. Huang, M. Larsson, P. Caroff, and H. Q. Xu, Nano Lett. 12, 6414 (2012).

[24] A. Das, Y. Ronen, Y. Most, Y. Oreg, M. Heiblum, and H. Shtrikman, Nat. Phys. 8, 887 (2012).

[25] L. P. Rokhinson, X. Liu, and J. K. Furdyna, Nat. Phys. 8, 795 (2012).

[26] S. Nadj-Perge, I. K. Drozdov, J. Li, H. Chen, S. Jeon, J. Seo, A. H. MacDonald, B. A. Bernevig, and A. Yazdani, Science 346, 602 (2014).

[27] A. Bühler, N. Lang, C. Kraus, G. Möller, S. Huber, and H. Büchler, Nat. Commun. 5, 5504 (2014).

[28] C.-K. Chiu, D. I. Pikulin, and M. Franz, Phys. Rev. B 91, 165402 (2015).

[29] K. Nomura, S. Ryu, M. Koshino, C. Mudry, and A. Furusaki, Phys. Rev. Lett. 100, 246806 (2008).

[30] Z. Ringel, Y. E. Kraus, and A. Stern, Phys. Rev. B 86, 045102 (2012).

[31] R. S. K. Mong, J. H. Bardarson, and J. E. Moore, Phys. Rev. Lett. 108, 076804 (2012).

[32] L. Fu and C. L. Kane, Phys. Rev. Lett. 109, 246605 (2012).

[33] P. Baireuther, J. M. Edge, I. C. Fulga, C. W. J. Beenakker, and J. Tworzydło, Phys. Rev. B 89, 035410 (2014).

[34] Y. Tanaka, Z. Ren, T. Sato, K. Nakayama, S. Souma, T. Takahashi, K. Segawa, and Y. Ando, Nat. Phys. 8, 800 (2012).

[35] P. Dziawa, B. J. Kowalski, K. Dybko, R. Buczko, A. Szczerbakow, M. Szot, E. Łusakowska, T. Balasubramanian, B. M. Wojek, M. H. Berntsen, O. Tjernberg, and T. Story, Nat. Mater. 11, 1023 (2012).
[36] S.-Y. Xu, C. Liu, N. Alidoust, M. Neupane, D. Qian, I. Belopolski, J. Denlinger, Y. Wang, H. Lin, L. Wray, G. Landolt, B. Slomski, J. Dil, A. Marcinkova, E. Morosan, Q. Gibson, R. Sankar, F. Chou, R. Cava, A. Bansil, and M. Hasan, Nat. Commun. 3, 1192 (2012).

[37] A. Y. Kitaev, Phys,-Uspekhi 44, 131 (2001).

[38] D. Wang, Z. Huang, and C. Wu, Phys. Rev. B 89, 174510 (2014).

[39] R. Wakatsuki, M. Ezawa, and N. Nagaosa, Phys. Rev. B 89, 174514 (2014).

[40] I. Seroussi, E. Berg, and Y. Oreg, Phys. Rev. B 89, 104523 (2014).

[41] I. C. Fulga, B. van Heck, J. M. Edge, and A. R. Akhmerov, Phys. Rev. B 89, 155424 (2014).

[42] M. Diez, I. C. Fulga, D. I. Pikulin, J. Tworzydło, and C. W. J. Beenakker, New J. Phys. 16, 063049 (2014).

[43] A. Rahmani, X. Zhu, M. Franz, and I. Affleck, arXiv:1504.05192.

[44] A. Rahmani, X. Zhu, M. Franz, and I. Affleck, arXiv:1505.03966.

[45] E. Cobanera, G. Ortiz, and Z. Nussinov, Phys. Rev. Lett. 104, 020402 (2010).

[46] P. Bak, Rep. Prog. Phys. 45, 587 (1982).

[47] W. Selke, Phys. Rep. 170, 213 (1988).

[48] F. Hassler and D. Schuricht, New J. Phys. 14, 125018 (2012).

[49] R. Thomale, S. Rachel, and P. Schmitteckert, Phys. Rev. B 88, 161103 (2013).

[50] The choice $\alpha>0$ can be made without loss of generality, since its sign can be changed by the gauge transformation $\gamma_{s} \rightarrow(-1)^{s} \gamma_{s}$ in Majorana language, or $\sigma_{s}^{x} \rightarrow-\sigma_{s}^{x}$ and $\sigma_{s}^{z} \rightarrow$ $(-1)^{s} \sigma_{s}^{z}$ in spin language.

[51] J. Haegeman, T. J. Osborne, and F. Verstraete, Phys. Rev. B 88, 075133 (2013).

[52] A. Milsted, J. Haegeman, T. J. Osborne, and F. Verstraete, Phys. Rev. B 88, 155116 (2013).

[53] A. Milsted, J. Haegeman, and T. J. Osborne, Phys. Rev. D 88, 085030 (2013).

[54] C. Holzhey, F. Larsen, and F. Wilczek, Nucl. Phys. B 424, 443 (1994).

[55] P. Calabrese and J. Cardy, J. Stat. Mech.: Theory Exper. (2004) P06002.

[56] L. Tagliacozzo, T. R. de Oliveira, S. Iblisdir, and J. I. Latorre, Phys. Rev. B 78, 024410 (2008).

[57] G. Refael and J. E. Moore, Phys. Rev. Lett. 93, 260602 (2004).

[58] D. Binosi, G. De Chiara, S. Montangero, and A. Recati, Phys. Rev. B 76, 140405 (2007).

[59] G. Refael and J. E. Moore, J. Phys. A: Math. Theor. 42, 504010 (2009).

[60] S. R. White, Phys. Rev. Lett. 69, 2863 (1992).

[61] J. A. Kjäll, M. P. Zaletel, R. S. K. Mong, J. H. Bardarson, and F. Pollmann, Phys. Rev. B 87, 235106 (2013). 